\title{
Reconstruction of the Denonvillier's fascia and posterior ligament of the external urethral sphincter: Assessment of its effect on urinary continence after laparoscopic radical prostatectomy
}

\author{
Pedro Sousa Passos ${ }^{1,2}$, Sara Teixeira Anacleto ${ }^{1}$, Rui Simeão Versos ${ }^{2}$, Mário Cerqueira Alves ${ }^{1}$, \\ Paulo Oliveira Mota ${ }^{1,3}$ \\ ${ }^{1}$ Department of Urology, Hospital de Braga, Portugal; \\ 2 Department of Urology, Hospital de Guimarães, Portugal; \\ ${ }^{3}$ Institute of Life and Health Sciences, University of Minho, Portugal.
}

\begin{abstract}
Summary Objectives: Some studies have shown that rhabdosphincter reconstruction provides an earlier return to continence after radical prostatectomy. We aim to study the impact of this procedure in urinary continence along with comparing two specific surgical techniques for posterior reconstruction.

Materials and methods: We studied a group of patients who were submitted to LRP with No Rhabdosphincter Reconstruction (NRR) and another group with Posterior Reconstruction of the Rhabdosphincter (PRR). The latter was further divided into two groups: "Rocco type stitch" group and "Bollens type stitch" group. We used three questionnaires (IIEF-5, ICIQ-SF and IPSS) to assess urinary continence and erectile function 90 days after surgery.

Results: Patients of PRR group had a better full continence rate than patients of NRR group at 90 days (96.6\% vs 33.3\%, $p<0.001)$. Concerning urinary incontinence $(p=0.116)$, lower urinary tract symptoms ( $p=0.543)$ and postoperative complication rates ( $p=0.738)$, our results suggested that there were no differences between the techniques studied.

Conclusions: Posterior reconstruction of the rhabdosphincter has significant benefits for urinary continence recovery on patients undergoing radical prostatectomy. No differences were observed in continence recovery between the two techniques analyzed. Additionally, reconstruction of the rhabdosphincter appears to be a safe procedure with no increased risk of postoperative complications.
\end{abstract}

KEY WORDS: Radical prostatectomy; Prostate cancer; Rhabdosphincter reconstruction; Postoperative complications; Erectile dysfunction; Urinary continence.

Submitted 11 June 2021; Accepted 9 July 2021

\section{INTRODUCTION}

One of the most common and effective treatments for localized Prostate Cancer ( $\mathrm{PCa}$ ) is the radical prostatectomy (RP) $(1,2)$. However, this procedure has shown to have a significantly negative impact on multiple quality-of-life domains due to its adverse effects such as urinary incontinence and erectile dysfunction $(3,4)$.
Previous studies show that urinary incontinence's prevalence widely varies from $2 \%$ to $65.5 \%$ and sexual dysfunction up to $87 \%(5,6)$.

Laparoscopic radical prostatectomy (LRP) has become a frequent treatment on local control of prostate cancer (8). Bollens et al. described an extraperitoneal laparoscopic surgical approach that combines the usual advantages of a laparoscopic procedure (less painful, reduced morbidity, earlier recovery) and the benefits of the open retropubic approach (avoid intraperitoneal organs injuries, potential risk of cancer spillage in the peritoneal cavity, intraperitoneal bleeding or urine leakage and allows possible later adjuvant radiotherapy) with results in terms of erectile function and continence equivalent to other techniques (9).

Prostate removal causes the destruction of the supporting system that anatomically and functionally separates the urethral sphincter complex from the prostatic apex and Denonvilliers' fascia, resulting in postoperative incontinence (6). The avoidance of these major complications after RP depends mainly on a high-quality surgical technique based on preservation, reconstruction, and reinforcement of the pelvis's anatomical structures, which will make a new supporting system after RP. Surgical techniques for posterior reconstruction of the rhabdosphincter were developed, namely Rocco Stitch (RS) and Bollens Stitch (BS) $(6,7,14)$.

A recent systematic review suggests that the rhabdosphincter's reconstruction could offer a significantly earlier return to continence in the first 30 days after RP still; its effect at 90 days remains controversial $(10,11)$. Salazar et al. (2019) concluded as well that the reconstruction of the rhabdosphincter is the only technique that has shown improved functional results through randomized trials (12).

This study compares the impact of posterior reconstruction of the rhabdosphincter on urinary continence recovery with no rhabdosphincter reconstruction after LRP procedure; while assessing, prospectively, two types of posterior reconstruction of the rhabdosphincter, namely RS or BS. 


\section{MATERIALS AND METHOdS}

\section{General}

We performed an observational and prospective nonrandomized study. The data was collected from the Braga's Hospital information system, Glintt ${ }^{\circledR}$, and via in-person interviews with patients, at the time of their postoperative appointment (90 days after LRP). All collected data were kept confidential.

The research protocol was submitted and approved by the Ethics Commission for Health of Braga's Hospital (CESHB) and by the Ethics Commission for Sciences of Life and Health of Minho's University (CEICVS). There were no potential conflicts of interest.

\section{Patient population}

The selected patients were older than 18 years old who attended Braga's Hospital consultations and submitted to LRP between January 2018 and November 2019.

The inclusion criteria were histological confirmation of PCa and localized PCa. The exclusion criteria included the presence of urinary incontinence before the procedure, previous radiation therapy of the prostate or pelvis, presence of prostatic surgery prior to the procedure, prior medical history of psychiatric disorder or drug addiction, and any other condition that contraindicated LRP. Patients with a history of urethral surgery, urethral stenosis or artificial urinary sphincter were also excluded.

According to these criteria, we selected a sample of 63 patients that had been submitted to LRP. Two different surgeons had performed the surgery in this group of patients, which was divided into two major groups:

NRR: retrospective group, whose patients have been submitted to LRP between January 2018 and December 2018 and had No Reconstruction of the Rhabdosphincter.

PRR: a group of patients who have been submitted to LRP, followed by Posterior Reconstruction of the Rhabdosphincter, between January 2019 and November 2019. According to the technique used for posterior rhabdosphincter reconstruction, this group was further divided into two sub-groups - RS and BS groups. Each technique was performed solemnly by a different surgeon (surgeon 1 - RS and surgeon 2 - BS).

Patients undergoing postoperative radiotherapy or major Clavien Dindo complications in the postoperative period were not included for the urinary continence assessment.

\section{Clinical data collection}

The demographic characteristics (age and sex), alcohol consumption, smoking habits, lower urinary tract symptoms, familiarity with $\mathrm{PCa}$, previous procedures of the urologic tract and number of pads used daily since LRP were asked to the patients under consultation.

The following data were collected by analyzing clinical reports: rhabdosphincter reconstruction (and type of stitch) or no reconstruction, patient's usual medication, metabolic disorders (hypertension, dyslipidemia, Diabetes mellitus, increased waist circumference), PSA ( $\mathrm{ng} / \mathrm{mL}$ ) previous to LRP, the result of digital rectal examination (normal or suspicious), prostate biopsy mode realization (aleatory, cognitive fusion or ultrasonography fusion) and associated complications, hospitalization duration and urinary catheter duration (days) after LRP and PSA value (ng/mL) one month after surgery (PSA TO). Clavien-Dindo Classification was usd to classify the complications rate after surgery. In this study, the pelvic floor rehabilitation protocols assessed were the Pelvic Floor Muscle Training (PFMT) in combination with behavioral therapy.

\section{Outcome measures}

The following outcome data were collected 90 days after the procedure by the healthcare provider:

- Urinary continence:

Declared urinary continence (defined by 0/1 safety pad per day) after physician evaluation on both the NRR and PRR groups, 90 days after LRP (33)

Number of pads/day used after LRP (RS vs BS)

International Consultation on Incontinence Questionnaire-

Short Form (ICIQ-SF) assessment (RS vs BS)

International Prostatic Symptoms Score (IPSS) assessment (RS vs BS).

- Erectile function:

Erectile function using International Index of Erectile Function (IIEF-5) assessment (RS vs BS).

- Morbidity associated to the surgical techniques:

Peri and postoperative complication rates (NRR vs PRR and RS vs BS).

Clavien-Dindo Classification (NRR vs PRR and RS vs BS).

\section{Questionnaires}

ICIQ-SF, validated in Portuguese (Cronbach's alpha coefficient of 0.88 ) to assess the patient's urinary continence (13).

IPSS, validated in Portuguese (Cronbach's alpha coefficient of 0.80$)$ to assess LUTS $(13,14)$.

IIEF-5, validated in Portuguese (Cronbach's alpha coefficient of 0.89) to assess erectile function (16).

\section{Statistical analysis}

The statistical analysis was performed with IBM SPSS ${ }^{\circledR}$ Statistics for Windows, Version 26.0.

Data normality was assessed through the Shapiro-Wilk test, skewness, kurtosis and visual evaluation of the histograms $(17,18)$.

To characterize the study's variables, we performed a descriptive analysis. Categorical variables were presented as frequencies (n) and proportions (\%). Numerical variables were presented as means (M) and standard deviations (SD) for symmetrically distributed variables, and medians (Mdn) and interquartile ranges (IQR) for non-symmetrically distributed variables.

The comparison of numerical variables between groups was performed with Student's T-test. The applied effect size measure was Cohen's d (19). A Mann-Whitney U test was performed when variables were not normally distributed. The effect size measure of this test was $r=Z / \sqrt{ }$, where ' $n$ ' is the total number of cases related to the study variable (20).

The Chi-Square test was used to compare proportions across qualitative variables. Fisher's exact test was used alternatively when the expected frequency was lower than 5 in more than $20 \%$ of the contingency table cells (21).

The applied effect size measure was phi $(\varphi)$ or Crammer's $\mathrm{V}$, since the cross-tables were two by two or three by two, respectively. 
To compare the urinary continence between-group NRR and PRR, we used a Chi-Square test $(\chi 2)$, and the effect size measure used was phi $(\varphi)$, since the cross-table was two by two. The Clavien-Dindo score was converted to a categorical variable: no complication (0), minor complication (I+II) and major complication $(\mathrm{III}+\mathrm{IV})$. A Fisher's exact test was used to compare these variables between groups NRR and PRR and to compare them between groups RS and BS. The effect size measure used was Cramer's V since the cross-tables were three by two.

For numerical variables, such as the number of pads/day used after LRP and the IIEF assessment results, ICIQ-SF assessment and IPSS assessment, we applied a MannWhitney U Test. This test was also used to compare these numerical variables between group RS and BS. The effect size measure used was $\mathrm{r}$.

A p-value of less than 0.05 was considered statistically significant, and the confidence interval was $95 \%$.

\section{RESULTS}

From the total sample population of 63 patients, 28 were not submitted to the posterior reconstruction of the rhabdosphincter (NRR), and 35 patients were (PRR).

The mean value for age was 64 years for both NRR and PRR group.

Table 1 shows the baseline characteristics between NRR and PRR groups, in which there were no differences. Table 2 shows the Gleason score and TNM staging of the included patients.

Regarding intraoperative complications, minor complications were reported in $\mathrm{n}=3(10.7 \%)$ from the NRR group and $n=6$ (17.2\%) from the PRR group; and major complications occurred in $\mathrm{n}=3(10.7 \%)$ and $\mathrm{n}=5(14.3 \%)$, in the NRR and PRR groups, respectively.

Complications were observed in both groups within days after surgery. However, after the mean (SD) follow-up period of 90 days, there were no complications in group PRR, and two patients from group NRR had complications. Regarding the Clavien Dindo classification, most patients did not present complications.

Table 3 compares postoperative medication and pelvic floor rehabilitation between NRR and PRR, and it shows no statistically significant differences. The table also shows that, at 90 days after surgery,

17 patients from NRR group and 14 from PRR group $(n=4(28.5 \%)$ and $\mathrm{n}=10(71.5 \%)$ from RS and BS groups, respectively) had already initiated PDH5 inhibitor ( $p=0.131)$; five patients from NRR and three from PRR had started PGEl ( $\mathrm{p}=$ $0.449)(n=3$ (100\%), from the RS group, and $\mathrm{n}=0$ from the BS group). Regarding pelvic floor rehabilitation, three patients (10.7\%) from NRR and two $(5,7 \%)$ from PRR had start-
Table 3.

Comparison of postoperative erectile dysfunction medication and pelvic floor rehabilitation between groups NRR and PRR.

\begin{tabular}{|c|c|c|c|c|c|}
\hline \multicolumn{2}{|l|}{ Phosphodiesterase 5 (PDH5) } & NR & PR & Statisti & p- \\
\hline \multirow[t]{2}{*}{ (2. } & $\mathrm{Ye}$ & 17 & 14 & \multirow{2}{*}{$? 2(1, n=63)$} & \multirow{2}{*}{0.13} \\
\hline & $\mathrm{N}$ & 11 & 21 & & \\
\hline \multirow{2}{*}{ Prostaglandin E1 Intracavernous ${ }^{a}$ (PGE } & Ye & 5 & 3 & \multirow{2}{*}{$n=2$} & \multirow{2}{*}{0.44} \\
\hline & $\mathrm{N}$ & 23 & 32 & & \\
\hline \multirow{2}{*}{$\begin{array}{l}\text { NRR: No Rhabdosp } \\
\text { Pelvic floor }\end{array}$} & $\mathrm{Ye}$ & 3 & 2 & \multirow{2}{*}{$n=2^{\text {er }}$} & \multirow{2}{*}{0.64} \\
\hline & $\mathrm{N}$ & 25 & 33 & & \\
\hline 1- Chi-square ?2); 2 - Fisher's exact test; a - $n$ & & & & & \\
\hline
\end{tabular}


Table 4.

Comparison of urinary continence between groups NRR and PRR.

\begin{tabular}{|c|c|c|c|c|c|}
\hline & & NRR & PRR & Statistics & p-value \\
\hline \multirow{2}{*}{ Urinary Incontinencé } & $\mathrm{Ye}$ & $16(66.7 \%$ & $1(3.4 \%)$ & \multirow{2}{*}{$x 2(1, n=54)=24.085$} & \multirow{2}{*}{$p<0.001$} \\
\hline & No & $8(33.3 \%)$ & $28(96.6 \%)$ & & \\
\hline \multicolumn{6}{|c|}{ 1-Chi-squaretest (2); a - n $\mathcal{C})$; Bold values denote statistical significance as $p<0.005$} \\
\hline
\end{tabular}

\section{Table 5.}

Baseline characteristics of RS and BS groups.

\begin{tabular}{|c|c|c|c|c|c|c|}
\hline & & RS n(\%) & BS n(\%) & Statistics & $p$-value & Effect size \\
\hline Age (years) & & $\begin{array}{c}64 \pm 6,093 \\
(n=25)\end{array}$ & $\begin{array}{c}64 \pm 7,, 291 \\
(n=10)\end{array}$ & $\begin{array}{c}t=-0,282 \\
(n=35)\end{array}$ & 0,78 & $d=0,149$ \\
\hline Family history of $\mathrm{PC}_{\hat{E}}$ & $\begin{array}{l}\text { Yes } \\
\text { No }\end{array}$ & $\begin{array}{c}1(4 \%) \\
24(96 \%)\end{array}$ & $\begin{array}{c}0(0 \%) \\
10(100 \%)\end{array}$ & $n=35^{2}$ & 1,000 & $\phi=-0,108$ \\
\hline Alcohol consumption & $\begin{array}{l}\text { Yes } \\
\text { No }\end{array}$ & $\begin{array}{l}3(12 \%) \\
22(88 \%)\end{array}$ & $\begin{array}{l}1(10 \%) \\
9(90 \%)\end{array}$ & $n=35^{2}$ & 1,000 & $\phi=-0,028$ \\
\hline Smoke & $\begin{array}{l}\text { Yes } \\
\text { No }\end{array}$ & $\begin{array}{l}8(32 \%) \\
17(68 \%)\end{array}$ & $\begin{array}{l}2(20 \%) \\
8(80 \%)\end{array}$ & $n=35^{2}$ & 0,686 & $\phi=-0,120$ \\
\hline Metabolic disorders & $\begin{array}{l}\text { Yes } \\
\text { No }\end{array}$ & $\begin{array}{l}10(40 \%) \\
15(60 \%)\end{array}$ & $\begin{array}{l}3(30 \%) \\
7(70 \%)\end{array}$ & $n=35^{2}$ & 0,709 & $\phi=-0,093$ \\
\hline
\end{tabular}

used more than one safety pad/day, but a significant difference was not found. The median score on ICIQ-SF was higher on RS than on BS (with no statistically significant differences, $\mathrm{p}=0.116$ ), and the median score on IIEF-5 assessment was 5 (0) in both groups.

Table 7 presents the impact of posterior rabdosphincter in both groups, RS and BS. After 90 days from LRP, no complications were observed in either group. Regarding Clavien Dindo classification, there were no complications in most of the subjects in both RS and BS group (68\% vs $70 \% ; p=0.738)$

\section{Discussion}

The results of this study suggest that posterior reconstruction of the rhabdosphincter improves the early continence of patients undergoing LRP, as it is described in

Table 6.

Comparison of the outcomes between RS and BS groups.

\begin{tabular}{|c|c|c|c|c|c|}
\hline & & RS & BS & Statistics & $p$-value \\
\hline Number Pacts/day & & 1(1) & $0(1)$ & $U=70.50, z=-107 / \pi=28$ & 0.282 \\
\hline Safety Pad & \begin{tabular}{|l|}
$0 / 1$ \\
$>1$ \\
\end{tabular} & \begin{tabular}{|l|}
$17(94.4 \%)$ \\
$1(5.6 \%)$ \\
\end{tabular} & $\begin{array}{l}10(100 \%) \\
0(0 \%)\end{array}$ & $n=28^{2}$ & 1000 \\
\hline IaQSF & & $9.5(11)$ & $4.5(10)$ & $U=57.50, Z=-15 / 3 n=28$ & 0.116 \\
\hline IPSS $^{a}$ & & $3(7)$ & $4(5)$ & $U=73.00, z=-0.609=27$ & 0.543 \\
\hline$\| E^{a}$ & & $5(0)$ & $5(0)$ & $U=7200, Z=-1952 \pi=28$ & 0.053 \\
\hline
\end{tabular}

\section{Table 7.}

Impact of posterior rabdosphincter in both groups, RS and BS.

\begin{tabular}{|c|c|c|c|c|c|c|}
\hline & & & RS & BS & STATISTICS & pVALUE \\
\hline Hospital Stay (days) & & & $3(2)$ & $3(2)$ & $U=100.5 ; Z=-0.94 ; \ln =35$ & 0.347 \\
\hline Urinary Catheter Duratio (days) & & & $13(5)$ & $14(12)$ & $U=104.5 ; Z=-0.760 n=35$ & 0.447 \\
\hline \multirow{6}{*}{ Complicati đas' Rocco Stitch; BS: Bol } & \multirow{2}{*}{$\leq 10$ days } & Yes & $2(8.0 \%)$ & $2(20 \%)$ & \multirow{2}{*}{$n=35^{2}$} & \multirow{2}{*}{0.561} \\
\hline & & No & $23(92 \%)$ & $8(80 \%)$ & & \\
\hline & \multirow{2}{*}{ ats-990:days } & Yes & $8(32 \%)$ & $3(30 \%)$ & \multirow{2}{*}{$N=35^{2}$} & \multirow{2}{*}{100} \\
\hline & & No & $17(68)$ & $7(70 \%)$ & & \\
\hline & \multirow{2}{*}{$>90$ days } & Yes & $0(0 \%)$ & $0(0 \%)$ & \multirow{2}{*}{$n=30^{2}$} & \\
\hline & & No & $20(100 \%)$ & $10(100 \%)$ & & \\
\hline \multirow{3}{*}{ CavienDind'b } & No Complication & & $17(68 \%)$ & $7(70 \%)$ & \multirow{3}{*}{$n=35^{2}$} & \multirow{3}{*}{0.738} \\
\hline & Minor & & $5(20 \%)$ & $1(10 \%)$ & & \\
\hline & Major & & $3(12 \%)$ & $2(20 \%)$ & & \\
\hline
\end{tabular}

several studies (11, 22-24). Rocco et al. (2007) conducted a prospective study on patients undergoing LRP, defining continence as no pads or one diaper/day. At 90 days after catheter removal, the continence rates were $92.3 \%$ on patients with posterior musculofascial plate reconstruction versus $76.9 \%$ on patients with no reconstruction (25). Rocco's study corroborates our results where patients of the PRR group had a better full continence rate than patients of the NRR group at 90 days.

Our study also showed no differences between the complication rates in the posterior rhabdosphincter reconstruction group and the non-reconstruction group and similar Clavien Dindo classification of complications. Coetho et al. (2011) related that overall complication rate and postoperative acute urinary retention rates at 30 days were similar between both groups (24). Grasso et al. (2016) have also shown no association between rhabdosphincter reconstruction and postoperative complications in a review and meta-analysis (11).

Regarding posterior rhabdosphincter reconstruction techniques (RS and BS), the current study found that both groups reported similar urinary continence rate and that one patient from RS group used more than one safety pad per day. In contrast, no pads were used in the BS group. For measuring urinary function, two questionnaires were applied to the reconstruction group: ICIQ-SF and IPSS, in which the median scores were similar in both groups. Machioka et al. (2019) demonstrated that the ICIQ-SF questionnaire was effective and convenient for evaluating urinary incontinence, including in patients after RP (26). However, this questionnaire is a subjective measure of the severity of urinary loss and the impact of urinary incontinence on quality-of-life (13), as its results depend on the patient's perspective. Since the results on IPSS are between 1 and 7 , lower urinary tract symptoms have a mild severity in these patients (27).

Assessing erectile function after 90 days from posterior rhabdosphincter reconstruction, the median score on IIEF5 assessment was the same in both groups. However, most patients were not on medication for erectile dysfunction at the time of the interview. Rocco et al. have described that erectile function was similar in reconstruction and nonreconstruction groups (28). Further literature corroborates that rhabdosphincter reconstruction techniques have no benefit for erectile function recovery (29).

The complication rates were similar in both RS and BS groups and, considering Clavien Dindo classification of complications, both groups had similar results. According to a meta-analysis stratified by surgical approaches, no association has been found between rhabdosphincter reconstruction and postoperative complications (11), suggesting that it is a safe procedure. Moreover, we measured selected demographic variables that are described in previous literature to have a great influence on postoperative urinary incontinence and erectile function (such as age, prostate size, PSA score, metabolic disorders, Gleason score, urinary catheter duration and surgical complications.) and 
found that there were no statistically significant differences between the groups NRR and PRR (3, 25, 30-32). This study has some limitations. The low sample size of the prospective part of the study $(\mathrm{n}=35)$ limits possible extrapolation. Additionally, the follow-up period (90days) was short for evaluating urinary continence, and the reference used for its definition in the PRR groups (number of pads/day) was a biased and subjective outcome, as opposed to a pad weight test. Furthermore, the non-reconstruction group is retrospective, and, therefore, the data were based on the physician interview and could have been affected by subjectivity.

Finally, several surgeons were responsible for performing the surgeries, which means that this was a non-controlled variable in this study.

More extensive research on surgical techniques for earlier urinary continence in LRP is required to allow more robust conclusions. Therefore, we recommend further studies on the current topic.

\section{Conclusions}

This study shows that the rhabdosphincter's posterior reconstruction has significant benefits for urinary continence recovery in the first 90 days on patients undergoing LRP.

Additionally, reconstruction of the rhabdosphincter appears to be a safe procedure with no increased risk of postoperative complications.

Concerning urinary continence and postoperative complication rates after LRP and postoperative complication rates, the results suggest that there are no statistically significant differences between Rocco Stitch and Bollens Stitch. Still, a system of support appears to play an essential role in urinary continence after surgery.

Further work is required to establish this.

\section{References}

1. Wein AJ, Kavoussi LR, Partin AW, et al. Campbell-Walsh Urology. 12th ed. Elsevier. 2020; 153:3529-3532.

2. Cao L, Yang Z, Qi L, Chen M. Robot-assisted and laparoscopic vs open radical prostatectomy in clinically localised prostate cancer. Medicine (Baltimore). 2019; 98:e15770.

3. Sanda MG, Dunn RL, Michalski J, et al. Quality of life and satisfaction with outcome among prostate-cancer survivors. N Engl J Med. 2008; 358:1250-61.

4. Miller DC, Saigal CS, Litwin MS. The demographic burden of urologic diseases in America. Urol Clin North Am. 2009; 36:11-27.

5. Trost L, Elliott DS. Male stress urinary incontinence: A review of surgical treatment options and outcomes. Adv Urol. 2012; 8:1-13.

6. Alivizatos G, Skolarikos A. Incontinence and erectile dysfunction following radical prostatectomy: a review. Scientific World Journal. 2005; 5:747-58.

7. Topaktas R, Urkmez A, Kutluhan MA, et al. Vesicourethral anastomosis including rhabdosphincter in retropubic radical prostatectomy: Technique and results. Arch Ital Urol Androl. 2019; 90:249-253.

8. Mottet N, Bellmunt J, Bolla $M$, et al. EAU-ESTRO-SIOG Guidelines on prostate cancer. Part 1: Screening, Diagnosis, and Local Treatment with Curative Intent. Eur Urol. 2017; 71:618-29.
9. Bollens R, Vanden Bossche M, Roumeguere T, et al. Extraperitoneal laparoscopic radical prostatectomy. Eur Urol. 2001; 40:65-9.

10. Rocco B, Cozzi G, Spinelli MG, et al. Posterior musculofascial reconstruction after radical prostatectomy: A systematic review of the literature. Eur Urol. 2012; 62:779-90.

11. Grasso AAC, Mistretta FA, Sandri M, et al. Posterior musculofascial reconstruction after radical prostatectomy: an updated systematic review and a meta-analysis. BJU Int. 2016; 118:20-34.

12. Salazar A, Regis L, Planas J, et al. Early continence after radical prostatectomy: A systematic review. Actas Urologicas Espanolas. 2019;43:526-535.

13. Tamanini TNJ, Dambros M, Levi ACD, et al. Validation of the "International Consultation on Incontinence Questionnaire-Short Form” (ICIQ-SF) for Portuguese. Rev Saude Publica. 2004; 38:438-44.

14. Mineo Bianchi F, Romagnoli D, D’Agostino D, et al. Posterior muscle-fascial reconstruction and knotless urethro-neo bladder anastomosis during robot-assisted radical cystectomy: Description of technique and its impacto $n$ urinary continence. Arch Ital Urol Androl. 2019; 91:5-10.

15. Berger M, Luz Junior PN, Brasil SN, Koff WJ. Statistical validation of the international prostatic symptom score (I-PSS) in portuguese. J Bras Urol. 1999; 25:225-34.

16. Pechorro PS, Calvinho AM, Pereira NM, Vieira RX. Validação da versão portuguesa do Índice Internacional de Função Eréctil-5 (IIEF-5). Rev Int Andrología. 2011; 9:3-9.

17. Razali NM, Wah YB. Power comparisons of Shapiro-Wilk, Kolmogorov-Smirnov, Lilliefors and Anderson-Darling tests. J Stat Model Anal. 2011; 2:21-33.

18. Kim HY. Statistical notes for clinical researchers: assessing normal distribution using skewness and kurtosis. Restor Dent Endod. 2013; 38:52.

19. Cohen J. Statistical power analysis for the behavioral sciences. $2^{\text {nd }}$ edition. New York: Lawrence Erlbaum Associates; 1988, Chapt 2, pp $20-52$.

20. Chan YH. Biostatistics 102: Quantitative data-parametric $\&$ non-parametric tests. Singapore Med J. 2003; 44:391-396.

21. Chan YH. Biostatistics 103: Qualitative data-tests of independence. Singapore Med J. 2003; 44:498-503.

22. Liao X, Qiao P, Tan Z, et al. "Total reconstruction" of the urethrovesical anastomosis contributes to early urinary continence in laparoscopic radical prostatectomy. Int Braz J Urol. 2016; 42:215-22.

23. Wu YP, Xu N, Wang ST, et al. The efficacy and feasibility of total reconstruction versus non total reconstruction of the pelvic floor on short-term and long-term urinary continence rates after radical prostatectomy: a meta-analysis. World J Surg Oncol. 2017; 20;15:228.

24. Coetho RF, Chauhan S, Orvieto MA, et al. Influence of modified posterior reconstruction of the rhabdosphincter on early recovery of continence and anastomotic leakage rates after robot-assisted radical prostatectomy. Eur Urol. 2011; 59:72-80.

25. Rocco B, Gregori A, Stener S, et al. Posterior Reconstruction of the rhabdosphincter allows a rapid recovery of continence after transperitoneal videolaparoscopic radical prostatectomy. Eur Urol. 2007; 51:996-1003.

26. Machioka K, Kadono Y, Naito R, et al. Evaluating urinary incontinence before and after radical prostatectomy using the international consultation on incontinence questionnaire-short form. Neurourol Urodyn. 2019; 38:726-33. 
27. Barry MJ, Fowler FJ, O'Leary MP, et al. The American urological association symptom index for benign prostatic hyperplasia. The Measurement Committee of the American Urological Association. J Urol. 1992; 148:1549-57.

28. Rocco F, Carmignani L, Acquati P, et al. Restoration of Posterior aspect of rhabdosphincter shortens continence time after radical retropubic prostatectomy. J Urol. 2006; 175:2201-6.

29. Tewari A, Jhaveri J, Rao S, et al. Total reconstruction of the vesico- urethral junction. BJU Int. 2008; 101:871-7.

30. Gershman B, Psutka SP, McGovern FJ, et al. Patient- reported functional outcomes following open, laparoscopic, and robotic assist- ed radical prostatectomy performed by high-volume surgeons at high-volume hospitals. Eur Urol Focus. 2016; 2:172-179.

31. Fujimoto M, Higuchi T, Kouichi H, Takada M. Association of statin use with storage lower urinary tract symptoms: Data mining of claims database. J Pharmacovigil. 2014; 2:5.

32. Heesakkers J, Farag F, Bauer RM, et al. Pathophysiology and contributing factors in postprostatectomy incontinence: A review. Eur Urol. 2017; 71: 936-44.

33. Kretschmer A, Hübner W, Sandhu JS, Bauer RM. Evaluation and management of postprostatectomy incontinence: a systematic review of current literature. Eur Urol Focus. 2016; 2:245-59.

\section{Correspondence}

Pedro Sousa Passos, MD (Corresponding Author)

pedrosousapassos@gmail.com

Department of Urology, Hospital de Braga

Largo Bairro do Jardim 3, 4900-467 Viana do Castelo (Portugal)

Sara Teixeira Anacleto, $M D$

sara.anacleto241@gmail.com

Mário Cerqueira Alves, MD

mcerqueiraalves@gmail.com

Paulo Oliveira Mota, MD

damota.paulo@gmail.com

Department of Urology, Hospital de Braga (Portugal)

Rui Simeão Versos, MD

rui.1971.versos@gmail.com

Department of Urology, Hospital de Guimarães (Portugal) 